\title{
3 Warum die Digitalisierung keine disruptiven Sprünge in der Gesundheitsversorgung auslöst, wohl aber eine Revolution der Versorgungsstrukturen zur Folge hat
}

\author{
Matthias Meierhofer
}

\section{Denn der Mensch bleibt Mensch: Analoger Heilungsprozess mit digitalen Versorgungsstrukturen}

Das Gesundheitswesen ist mit anderen Wirtschaftszweigen, beispielsweise der Automobilindustrie, nicht zu vergleichen. Anders als in anderen Branchen ist das ,produzierte" Gut - Gesundheit - immer aufs engste verbunden mit dem Menschen. Aus diesem Grund können wir im Gesundheitswesen nicht von disruptiven Veränderungen aufgrund der zunehmenden Digitalisierung sprechen. Der Mensch wird durch seine Biologie immer selbstgesteuert sein. Sein Körper ist nicht digitalisierbar und seine Gesundheit somit immer Ergebnis einer analogen Lebensweise beziehungsweise eines analogen Heilungsprozesses. Dieser Heilungsprozess wird jedoch begleitet von einem Gesundheitssystem, das sich gerade in einem massiven Wandel befindet.

Dieser Wandel basiert auf der zunehmenden Digitalisierung, die enormen Einfluss auf die Strukturen, die Protagonisten sowie die Art und Qualität der Leistungen des Gesundheitssystems hat. Damit gehen Chancen einher, das Gesundheitswesen von Grund auf zu modernisieren, Patienten stärker in die Prozesse einzubinden, finanzielle und personelle Engpässe auszugleichen und Versorgungslücken zu schließen. Um diese Chancen zu nutzen, müssen tradierte Strukturen jedoch verändert und Versorgungsprozesse neu gedacht werden. Insofern steht auch der Medizin durch die Digitalisierung eine Revolution bevor und es ist weniger eine technologische denn eine philosophisch/konzeptionelle Frage, inwieweit diese tatsächliche Mehrwerte für die medizinische Versorgung von morgen mit sich bringt. 
3 Warum die Digitalisierung keine disruptiven Sprünge in der Gesundheitsversorgung auslöst, wohl aber eine Revolution der Versorgungsstrukturen zur Folge hat

\title{
Digitale Revolution: Was kann, was muss und was darf nicht passieren?
}

\author{
Ansatzpunkte für die Neugestaltung einer digitalen Gesundheitsver- \\ sorgung sind:
}
vernetzung von Patienten, Leistungserbringern und Kosten- trägern
- aktive Steuerung der Versorgung mithilfe einer zentralen Gesundheitsakte
- Nutzung des digitalen Datenpools

Die Vernetzung der Protagonisten in der Gesundheitsversorgung ist technologisch einfach umzusetzen. Über eine zentrale Infrastruktur, zum Beispiel die mit dem EHealth-Gesetz angestoßene Telematikinfrastruktur (TI), wird eindeutig definiert, welche Informationen in welchem Format in einer zentralen Akte landen, auf die der Patient, aber auch Leistungserbringer und Kostenträger Zugriff haben. Dass diese Umstellung Zeit braucht und im Vergleich zu anderen Ländern und Branchen sehr schleppend vorangeht, liegt an der eigentlichen Herausforderung bei dieser Transformation: Zwischen allen Protagonisten müssen die bestehenden Beziehungen, ebenso wie gewohnte Privilegien und Pflichten neu definieren werden.

Außerdem stehen Leistungserbringer und Krankenkassen vor der Herausforderung, den gewohnten Versorgungsprozess an die veränderten Bedürfnisse der Patienten und an die neuen Möglichkeiten der medizinischen Versorgung anzupassen (Beispiel Telemedizin). Dazu müssen einzelne Leistungsschritte und Vergütungsmodelle festgelegt werden. Auch an dieser Stelle ist ein generelles Umdenken notwendig, zu dem vielfach schlicht der Mut fehlt.

Ein weiterer Themenkomplex, der mit der Digitalisierung einhergeht, ist der Informationsgewinn. Dank digitaler Technologien werden im großen Stil Gesundheitsdaten gewonnen, die wiederum Erkenntnisse liefern, aus denen sich bessere Diagnoseverfahren und neue Therapien ableiten lassen. Das wird Krankheitsverläufe verändern, zu neuen Spezialisierungen und Fähigkeiten beim medizinischen Fachpersonal führen und die Qualität der Versorgung verbessern. Auch sind Leistungserbringer und Kostenträger mithilfe der Daten in der Lage, Versorgungsangebote gezielt zu steuern, Angebote auf die Bedürfnisse der Patienten zuzuschneiden und sich zu spezialisieren. Letztendlich kann die Transparenz über Leistungen dazu führen, eine an Qualität und Wirtschaftlichkeit orientierte Vergütung zu etablieren.

Doch: Wer nicht bereit ist, von gewohnten Versorgungsprozessen abzuweichen, wird die Fülle der Möglichkeiten, die die Digitalisierung bietet, nicht für sich nutzen können und am Ende aus der Gesundheitsversorgung ausscheiden. Führt der Unwille beim Einsatz digitaler Technologien heute noch lediglich zu einem Stillstand in der Versorgungentwicklung, bedeutet er morgen das Aus einer qualitativ hochwertigen Gesundheitsversorgung. 


\title{
Chancen und Risiken: Wie schaffen wir Vernetzung und eine digitale Akte?
}

\author{
Heutige Hürden der Digitalisierung: \\ - strenger Datenschutz \\ - keine Konzepte zur IT-Sicherheit \\ - fehlende finanzielle Mittel
}

\begin{abstract}
Doch sind das wirklich die Gründe, warum Deutschland in Sachen digitaler Versorgungsstrukturen manchem Nachbarstaat hinterherhinkt? Klar ist: Mit dem Einsatz neuer Technologien wird vieles anders - und das gefällt längst nicht jedem. Deutschland tut sich mitunter extrem schwer, die notwendigen Schritte zu gehen und Gesetze an die neue Situation anzupassen. Die Gründe hierfür liegen zum Beispiel in einem ausgeprägtem Bestandsdenken der Beteiligten und einer generellen Skepsis gegenüber der Digitalisierung (Stichwort Datenschutz). Doch wenn klare politische Vorgaben fehlen, führt das zu Unsicherheit bei den Leistungserbringern und der Industrie, die in der Folge auf die Innovationsbremse treten. Mit dem E-Health-Gesetz wurde ein erster Rahmen geschaffen, mehr Transparenz und Verbindlichkeit in den Digitalisierungsprozess zu bringen. Was nach wie vor fehlt, sind klare Vorgaben zum Datenschutz und zur IT-Sicherheit, die es noch zu definieren gilt.

Oft wird auch die vergleichsweise schlechte Finanzierungssituation in Gesundheitseinrichtungen als Grund für die schleppende Umsetzung digitaler Projekte angeführt. Fehlende Investitionen in der IT seien der Grund dafür, dass das deutsche Gesundheitssystem in großen Teilen papierbasiert ist. Das ist aber nur ein Teil der Wahrheit. Der andere ist, dass es neben den finanziellen Mitteln an konkreten Strategien und Konzepten für eine durchgehende Digitalisierung mangelt. Die erfordert nämlich nicht nur die Implementierung von IT-Systemen, sondern auch die Änderung von Arbeitsprozessen, beispielsweise die Einbindung der Patienten oder die einrichtungsund sektorenübergreifende Behandlung.
\end{abstract}

\section{Die Digitalisierung der Gesundheitsversorgung geht von Krankenhäusern aus}

Der Wandel in der deutschen Gesundheitsversorgung durch die Digitalisierung beginnt primär in den Krankenhäusern. Denn hier sind die Anforderungen des Systems - Dokumentationspflichten, Anforderungen an die Patientensicherheit, fehlendes Fachpersonal, finanzieller Druck - am deutlichsten spürbar und wirtschaftlich relevant. Rund ein Drittel der deutschen Krankenhäuser schrieb 2016 rote Zahlen. Ohne Veränderungen werden diese Häuser künftig nicht mehr an der Gesundheitsversorgung teilnehmen.

Die Digitalisierung bietet die Möglichkeit, den Evolutionsprozess im Krankenhaus massiv zu beschleunigen. Ob bei der Vernetzung von ambulanten und stationären Strukturen, in der Qualitätssicherung oder in der Unterstützung der Pflege, aber auch 
3 Warum die Digitalisierung keine disruptiven Sprünge in der Gesundheitsversorgung auslöst, wohl aber eine Revolution der Versorgungsstrukturen zur Folge hat

in der Transparenz gegenüber den Patienten - die Digitalisierung bietet Lösungswege für die drängendsten Fragen und besitzt das Potenzial, immensen Nutzen für alle Beteiligten nach sich zu ziehen. Kein Wunder also, dass die IT in Krankenhäusern mittlerweile als Managementthema in den Chefetagen angekommen ist. Vorreiter sind hier die Universitätskliniken sowie große Klinikketten, bei denen die strategische Digitalisierung der Prozesse höchste Priorität genießt, beispielsweise in Form der Einführung digitaler Patientenakten und dem Abbau papierbasierter Dokumentationen.

Der Grund für dieses Engagement liegt auf der Hand: Stationäre Einrichtungen befinden sich im Wettbewerb, unterliegen einem Spezialisierungsdruck und buhlen um die Gunst der Patienten. Darum müssen Prozesse effizient gestaltet werden, um Mitarbeiter zu entlasten, damit diese mehr Zeit für Patienten haben. Abteilungen müssen interdisziplinär aufgestellt sein, was eine Vernetzung erfordert, die nur ITseitig gestaltet werden kann. Und Patienten müssen stärker als bisher in die Versorgung eingebunden werden, beispielsweise durch das zur Verfügung stellen von Online-Services oder der Patientenakte.

Um diesen Anforderungen gerecht zu werden, müssen Kliniken und Industrie Hand in Hand arbeiten, um praxisnahe IT-Lösungen zu schaffen, die sektorenübergreifend einzelne Stufen im Versorgungsprozess orchestrieren und den Fokus auf das Wohl des Patienten setzen.

Dieses Patientenwohl wird in erster Linie durch den hürdenlosen Übergang zwischen den Sektoren geschaffen, also zwischen „Besuch beim Haus-/Facharzt“, „Krankenhausaufenthalt" und „Reha/Nachbehandlung“. Die Digitalisierung leistet der Erreichung dieses Ziels Vorschub, weil beispielsweise eine Vernetzung für eine höhere Verfügbarkeit und auch eine Konsolidierung notwendiger medizinischer Informationen sorgt. In diesem Zusammenhang muss auch der sogenannte zweite Gesundheitsmarkt, also das Angebot, das sich parallel zu den Kassenleistungen beispielsweise durch Smartphone-Apps, entwickelt hat, in den Kreislauf eingebunden werden.

Konkret: Wenn der Patienten im Vorfeld zu einer geplanten OP online auf die Aufklärungsformulare zugreifen kann, Informationen erhält, die ihm dazu verhelfen, bereits den Aufnahmefragebogen auszufüllen, dann ist bei seinem Besuch im Krankenhaus die Anamnese bereits erfolgt, der Patienten weiß, was ihn erwartet, das Krankenhauspersonal ist entlastet und kann sich um den Patienten kümmern. Bereits während seines Krankenhausaufenthalts stellen die behandelnden Ärzte die Dokumentation zur OP, Therapie und Genesung in der Patientenakte all denjenigen zur Verfügung, die an der Rehabilitation und Nachbehandlung beteiligt sind. Der Patient profitiert von maximal informierten Behandlern und wird mit Hilfe digitaler Technologien über die Art seiner Erkrankung, richtige Verhaltensweisen sowie Behandlungsanweisungen informiert. So wird die Digitalisierung in der Gesundheitsversorgung am Ende auch den Heilungsprozess positiv beeinflussen, ohne ihn dabei disruptiv zu verändern. 